\title{
Some Equivalences and Dualities via Static Modules
}

\author{
Salah Al-Nofayee ${ }^{1}$, Syed Khalid Nauman ${ }^{2}$ \\ ${ }^{1}$ Department of Mathematics, Taif University, Taif, Saudi Arabia \\ ${ }^{2}$ Department of Mathematics, King Abdulaziz University, Jeddah, Saudi Arabia \\ E-mail: \{alnofayee, synakhaled\}@hotmail.com \\ Received July 14, 2011; revised August 16, 2011; accepted August 25, 2011
}

\begin{abstract}
For a ring $A$, an extension ring $B$, a fixed right $A$-module $M$, the endomorphism ring $D$ formed by $M$, the endomorphism ring $E$ formed by $M \otimes_{A} B$, and the endomorphism ring $F$ formed by $\operatorname{Hom}_{A}(B, M)$, we present equivalences and dualities between subcategories of $B$-modules which are finitely cogenerated injective as $A$-modules and $E$-modules and $F$-modules which are finitely generated projective as $D$-modules.
\end{abstract}

Keywords: Static Modules, Finitely Cogenerated Injective Modules, Finitely Generated Projective Modules

\section{Introduction}

Let $A$ be any ring, $M$ a fixed right $A$-module and $D=\operatorname{End}_{A}(M)$. An object $V$ in $M o d-A$ (respectively $W$ in $M o d-D$ ) is said to be $M$-static (respectively $M$-adstatic), in case $V$ remains invariant under the composite covariant functor $\operatorname{Hom}_{A}(M,-) \otimes_{D} M$ (respectively

$\operatorname{Hom}_{A}\left(M,-\otimes_{D} M\right)$. We denote by $\operatorname{Stat}(M)$ and $\operatorname{Adst}(M)$ the classes of all static and adstatic objects of Mod-A and $M o d-D$, respectively. We will use the notation $F C I-A$ and $F G P-D(D-F G P)$ for the classes of all finitely cogenerated injective and finitely generated projective objects in Mod-A, Mod-D (D-Mod) respectively.

It is clear that

$$
\operatorname{Hom}_{A}(M,-): \operatorname{Stat}(M) \rightleftarrows \operatorname{Adstat}(M):-\bigotimes_{D} M
$$

is an equivalence and a special case of this equivalence is an equivalence between $\operatorname{Mod}(A$ :weak $M)$ and $\operatorname{Mod}(D$ : weak $D)$ where $\operatorname{Mod}(A$ :weak $M)$ is the full additive subcategory of all those objects which weakly divide (i.e. divide some finite direct sum of copies of) $M$ in $M o d-A$ and $\operatorname{Mod}(D$ :weak $D)=F G P-D$.

In [1], which is an extension of the work of Xue in [2], it is proved that $M$ is a finitely cogenerated injective cogenerator of $\operatorname{Mod}-A$ iff $\operatorname{Mod}(A$ :weak $M)=F C I-A$ and that this fact is equivalent to the existence of an equivalence or a duality between $F C I-A$ and $F G P-D$ or FCI-A and $D-F G P$, respectively.

Let $B$ be another ring and $\alpha: A \rightarrow B$ a ring homomorphism. Suppose that $E=\operatorname{End}_{B}\left(M \otimes_{A} B\right)$. Then the ring homomorphism $\sigma: D \rightarrow E$ defined via

$\sigma(d)(m \otimes b)=d(m) \otimes b$, for all $(d, m, b) \in D \times M \times B$ is clearly identity preserving. Similarly if

$F=\operatorname{End}_{B}\left(\operatorname{Hom}_{A}(B, M)\right)$, then the ring homomorphism $\alpha: D \rightarrow F$ defined via $\alpha(\mathrm{d})(f)=d \circ f$, for all $d \in D$ and $f \in \operatorname{Hom}_{A}(B, M)$, is clearly identity preserving.

Let us set

$\operatorname{Mod}(B:$ weak $M)=\left\{V \in \operatorname{Mod}-B: V_{A}\right.$ weakly divides $M$ in Mod-A\},

$\operatorname{Mod}(B: F C I-A)=\left\{V \in M o d-B: V_{A} \in F C I-A\right\}$,

$\operatorname{Mod}(E: F G P-D)=\left\{W \in M o d-E: W_{D} \in F G P-D\right\}$,

$\operatorname{Mod}(E: D-F G P)=\left\{W \in E-M o d: W_{D} \in D-F G P\right\}$,

$\operatorname{Mod}(F: F G P-D)=\left\{W \in \operatorname{Mod}-F: W_{D} \in F G P-D\right\}$,

$\operatorname{Mod}(F: D-F G P)=\left\{W \in F-M o d: W_{D} \in D-F G P\right\}$.

With the assumption that $M \otimes_{A} B$ is $M$-static in $\operatorname{Mod}-A$, an equivalence between subcategories $\operatorname{Mod}(B$ : weak $M)$ and $\operatorname{Mod}(E: F G P-D)$ of $M o d-B$ and $M o d-E$, respectively, is established in [3]. This in fact is a generalization of the work of Cline [4] and Dade [5] on stable Clifford theory. In this work using the same assumption it is proved that $\operatorname{Mod}(B: F C I-A)$ and $\operatorname{Mod}(E: F G P-D)$ are equivalent and with some additional assumption dualities between $\operatorname{Mod}(B: F C I-A)$ and both $\operatorname{Mod}(F: D-F G P)$ and $\operatorname{Mod}(E: D-F G P)$ are deduced.

We assume that the rings are associative with identity, the ring homomorphisms are identity preserving, all (left, right) modules are unital, and all subcategories are full and additive.

\section{Equivalences and Dualities}

We fix here all the notations and terminology from the previous section.

The following Theorem is proved in [1], Theorem 3. 
Theorem 2.1 The following statements are equivalent for a right $A$-module $M$.

1) $M$ is a finitely cogenerated injective cogenerator in Mod-A,

2) $\operatorname{Mod}-(A$ weak $M)=F C I-A$,

3) $\mathrm{Hom}_{A}(M,-): F C I-A \rightleftarrows F G P-D:-\otimes_{D} M$ define an equivalence,

4) $\operatorname{Hom}_{A}(-, M): F C I-A \rightleftarrows D-F G P: \operatorname{Hom}_{D}(-, M)$ define a duality.

Corollary 2.2 Let $M$ be a right A-module. If $M$ is a finitely cogenerated injective cogenerator in Mod- $A$, then $\operatorname{Mod}(B$ :weak $M)=\operatorname{Mod}(B: F C I-A)$.

Proof. By Theorem 2.1-(2) and the definitions of the subcategories $\operatorname{Mod}(B: F C I-A)$ and $\operatorname{Mod}(B:$ weak $M)$, the result follows.

Remark 2.3 From now on we assume that $M \otimes_{A} B$ is $M$-static as an A-module. With this assumption, one can deduce that $M \otimes_{A} B \cong E \otimes_{D} M$ in $D-M o d-A$. The details of internal maps of this isomorphism and their proofs can be seen in [3].

The following Theorem is proved in [3], Theorem 5.5.

Theorem 2.4 For a right $A$-module $M$, the restrictions of the additive functors

$$
\operatorname{Hom}_{B}\left(M \otimes_{A} B,-\right) \text { and }-\otimes_{E}\left(M \otimes_{A} B\right)
$$

form an equivalence of the full additive subcategories $\operatorname{Mod}(B$ :weak $M)$ and $\operatorname{Mod}(E: F G P-D)$ of Mod-B and $M o d-E$, respectively.

Proposition 2.5 For a right A-module $M$, the following statements are equivalent.

1) $\operatorname{Mod}(B:$ weak $M)=\operatorname{Mod}(B: F C I-A)$,

2) The restrictions of the additive functors

$\operatorname{Hom}_{B}\left(M \otimes_{A} B,-\right)$ and $-\otimes_{E}\left(M \otimes_{A} B\right)$ form an equivalence of the full additive subcategories $\operatorname{Mod}(B: F C I-A)$ and $\operatorname{Mod}(E: F G P-D)$ of Mod-B and Mod-E, respectively.

Proof. $(1) \Rightarrow(2)$ By Theorem 2.4.

$(2) \Rightarrow(1)$ By Theorem 2.4, it is clear that

$$
\begin{aligned}
\operatorname{Mod}(B: \text { weak } M) & =\operatorname{Im}\left(-\left.\otimes_{E}\left(M \otimes_{A} B\right)\right|_{\operatorname{Mod}(E: F G P-D)}\right) \\
& =\operatorname{Mod}(B: F C I-A)
\end{aligned}
$$

Remark 2.6 Recall that $E=\operatorname{End}_{B}\left(M \otimes_{A} B\right)$ and $F=\operatorname{End}_{B}\left(\operatorname{Hom}_{A}(B, M)\right)$

1) For the following one can see [6], Lemma 3.2. We have

$$
\operatorname{Hom}_{A}\left(\operatorname{Hom}_{A}(B, M), M\right) \cong F,
$$

and in this case

$$
\begin{gathered}
\operatorname{Hom}_{D}\left(\operatorname{Hom}_{A}\left(\operatorname{Hom}_{A}(B, M), M\right), M\right) \cong \operatorname{Hom}_{D}(F, M) \\
\text { So if } \operatorname{Hom}_{A}(B, M) \text { is M-reflexive, then } \\
\operatorname{Hom}_{A}(B, M) \cong \operatorname{Hom}_{D}(F, M)
\end{gathered}
$$

therefore

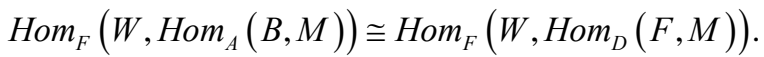

But by the adjoint associativity theorem we have

$$
\operatorname{Hom}_{F}\left(W, \operatorname{Hom}_{D}(F, M)\right) \cong \operatorname{Hom}_{D}(W, M)
$$

thus

$$
\operatorname{Hom}_{F}\left(W, \operatorname{Hom}_{A}(B, M)\right) \cong \operatorname{Hom}_{D}(W, M)
$$

2) Suppose that $\left(M \otimes_{A} B\right) \cong \operatorname{Hom}_{A}(B, M)$ as B-modules and hence as A-modules. Then we have the following sequence of D-isomorphisms:

$$
\begin{aligned}
E & \cong \operatorname{Hom}_{B}\left(\left(M \otimes_{A} B\right),\left(M \otimes_{A} B\right)\right) \\
& \cong \operatorname{Hom}_{B}\left(\left(M \otimes_{A} B\right), \operatorname{Hom}_{A}(B, M)\right) \\
& \cong \operatorname{Hom}_{A}\left(\left(M \otimes_{A} B\right) \otimes_{B} B, M\right) \\
& \cong \operatorname{Hom}_{A}\left(\left(M \otimes_{A} B\right), M\right) .
\end{aligned}
$$

This means that

$$
\operatorname{Hom}_{D}(E, M) \cong \operatorname{Hom}_{D}\left(\operatorname{Hom}_{A}\left(\left(M \otimes_{A} B\right), M\right), M\right)
$$

in E-Mod-A. Further, if $\left(M \otimes_{A} B\right)$ is M-reflexive, then

$$
\operatorname{Hom}_{D}(E, M) \cong M \otimes_{A} B \cong E \otimes_{D} M
$$

in $E$-Mod-A.

3) On the other hand, according to the assumption that $\left(M \otimes_{A} B\right) \cong \operatorname{Hom}_{A}(B, M)$, it is easy to see that $E \cong F$ in D-Mod.

The following Proposition is proved in [6, Proposition 3.3].

Proposition 2.7 Let $M$ be a right A-module. If $\operatorname{Hom}_{A}(B, M)$-reflexive is $M$-reflexive, then

1) $V_{B}$ is $\operatorname{Hom}_{A}(B, M)$-reflexive if and only if $V_{A}$ is $M$-reflexive.

2) ${ }_{F} W$ is $\operatorname{Hom}_{A}(B, M)$-reflexive if and only if ${ }_{D} W$ is M-reflexive.

Theorem 2.8 Let $M$ be a right A-module. Let $\operatorname{Hom}_{A}(B, M)$ be M-reflexive. Then the restrictions of the additive functors $\operatorname{Hom}_{B}\left(-, \operatorname{Hom}_{A}(B, M)\right)$ and $\operatorname{Hom}_{F}\left(-, \operatorname{Hom}_{A}(B, M)\right)$ form a duality of the full additive subcategories $\operatorname{Mod}(B$ :weak $M)$ and $\operatorname{Mod}(F: D-F G P)$ of Mod-B and F-Mod, respectively.

Proof. Let $V \in \operatorname{Mod}(B$ :weak $M)$, hence $V \in \operatorname{Mod}(A$ : weak $M$ ). This means that $M^{n} \cong V \oplus U$, for some $U$ and some positive integer $n$. Now we have

$$
D^{n} \cong\left(M^{n}\right)^{*} \cong V^{*} \oplus U^{*},
$$

where $(-)^{*}=\operatorname{Hom}_{A}(-, M)$. Thus $V^{*}=\operatorname{Hom}_{A}(V, M)$ $\in D-F G P$. We have the following isomorphisms,

$$
\begin{aligned}
\operatorname{Hom}_{B}\left(V, \operatorname{Hom}_{A}(B, M)\right) & \cong \operatorname{Hom}_{A}\left(\left(V \otimes_{B} B\right), M\right) \\
& \cong \operatorname{Hom}_{A}(V, M)
\end{aligned}
$$


Hence $\operatorname{Hom}_{B}\left(V, \operatorname{Hom}_{A}(B, M)\right) \in D-F G P$ and so belongs to $\operatorname{Mod}(F: D-F G P)$. Let $W \in \operatorname{Mod}(F: D-F G P)$. Then $W \in D$ - $F G P$ and so $D^{n} \cong W \oplus Q$, for some $Q$ in $D$-Mod and some positive integer $n$. Now we have

$$
W^{*} \oplus Q^{*} \cong\left(D^{n}\right)^{*} \cong M^{n},
$$

where $(-)^{*}=\operatorname{Hom}_{D}(-, M)$. Therefore $W^{*}=\operatorname{Hom}_{D}(W, M)$ weakly divides $M$ in $M o d-A$. i.e. $W^{*} \in \operatorname{Mod}(A$ :weak $M)$ and hence it belongs to $\operatorname{Mod}(B$ : weak $M$ ). Now as we can see from Remark 2.6-(1),

$$
\operatorname{Hom}_{F}\left(W, \operatorname{Hom}_{A}(B, M)\right) \cong \operatorname{Hom}_{D}(W, M)
$$

We deduce that $\operatorname{Hom}_{F}\left(W, \operatorname{Hom}_{A}(B, M)\right) \in \operatorname{Mod}(B$ : weak $M$ ).

According to the fact that $M^{n}$ and $D^{n}$ are $M$-reflexive, it is clear that for every $V$ in $\operatorname{Mod}(A$ :weak $M), V$ is $M$-reflexive and for every $W$ in $\mathrm{D}-F G P, W$ is $M$-reflexive. Applying Proposition 2.7, since $\operatorname{Hom}_{A}(B, M)$ is $M$ reflexive, every $V$ in $\operatorname{Mod}(B$ :weak $M)=\operatorname{Mod}(B: F C I-A)$ is $H m_{A}(B, M)$-reflexive, and every $W$ in $\operatorname{Mod}(F: D-F G P)$ is $\operatorname{Hom}_{A}(B, M)$-reflexive.

Theorem 2.9 Let $M$ be a right A-module. Let $\operatorname{Hom}_{A}(B, M)$ be M-reflexive. Then the following statements are equivalent for $M$.

1) $\operatorname{Mod}(B:$ weak $M)=\operatorname{Mod}(B: F C I-A)$,

2) The restrictions of the additive functors $\operatorname{Hom}_{B}\left(-, \operatorname{Hom}_{A}(B, M)\right)$ and $\operatorname{Hom}_{F}\left(-, \operatorname{Hom}_{A}(B, M)\right)$ form a duality of the full additive subcategories Mod (B:FCI-A) and Mod(F:D-FGP) of Mod-B and F-Mod, respectively.

Proof. (1) $\Rightarrow$ (2) By Theorem 2.8.

$(2) \Rightarrow(1)$ Consider the following isomorphisms obtained by the adjoint associativity theorem and Remark 2.6, we have

$$
\operatorname{Hom}_{B}\left(V, \operatorname{Hom}_{A}(B, M)\right) \cong \operatorname{Hom}_{A}(V, M)
$$

and

$$
\operatorname{Hom}_{F}\left(W, \operatorname{Hom}_{A}(B, M)\right) \cong \operatorname{Hom}_{D}(W, M)
$$

Let $V \in \operatorname{Mod}(B: F C I-A)$, then $V^{*}=\operatorname{Hom}_{B}\left(V, \operatorname{Hom}_{A}(B, M)\right) \in \operatorname{Mod}(F: D-F G P)$.

So $D^{n} \cong V^{*} \oplus Q$, for some $Q$ and some positive integer $n$. Now we have the following sequence of isomorphisms,

$$
\begin{aligned}
M^{n} & \cong \operatorname{Hom}_{D}\left(D^{n}, M\right) \\
& \cong \operatorname{Hom}_{D}\left(V^{*} \oplus Q, M\right) \\
& \cong \operatorname{Hom}_{D}\left(V^{*}, M\right) \oplus \operatorname{Hom}_{D}(Q, M) \\
& \cong \operatorname{Hom}_{F}\left(V^{*}, \operatorname{Hom}_{A}(B, M)\right) \oplus \operatorname{Hom}_{D}(Q, M) \\
& \cong V \oplus \operatorname{Hom}_{D}(Q, M),
\end{aligned}
$$

where the fourth isomorphism is due to (2) and the fifth isomorphism is due to the fact that $V$ is

$H_{A}(B, M)$-reflexive. So $V \in \operatorname{Mod}(A$ :weak $M)$ and therefor it belongs to $\operatorname{Mod}(B$ :weak $M)$.

Conversely suppose that $V \in \operatorname{Mod}(B$ :weak $M)$. This means that $M^{n} \cong V \oplus U$, for some $U$ and some positive integer $n$. Now we have the following isomorphisms,

$$
\begin{aligned}
D^{n} & \cong \operatorname{Hom}_{A}\left(M^{n}, M\right) \\
& \cong \operatorname{Hom}_{A}(V \oplus U, M) \\
& \cong \operatorname{Hom}_{A}(V, M) \oplus \operatorname{Hom}_{A}(U, M)
\end{aligned}
$$

Thus $\operatorname{Hom}_{A}(V, M) \in D-F G P$. By (1) it is clear that

$$
V^{*}=\operatorname{Hom}_{B}\left(V, \operatorname{Hom}_{A}(B, M)\right) \in \operatorname{Mod}(F: D-F G P) .
$$

Therefore by the given duality

$$
\operatorname{Hom}_{F}\left(V^{*}, \operatorname{Hom}_{A}(B, M)\right) \in \operatorname{Mod}(B: F C I-A) .
$$

Now since $V \in \operatorname{Mod}(A$ :weak $M)$, it is $M$-reflexive, so by Proposition 2.7 it is $\operatorname{Hom}_{A}(B, M)$-reflexive. Hence the result follows by (3).

Theorem 2.10 Let $M$ be a right A-module. Let $\left(M \otimes_{A} B\right) \cong \operatorname{Hom}_{A}(B, M)$ as B-modules and let $M \otimes_{A} B$ be $M$-reflexive. Then the following statements are equivalent for $M$.

1) $\operatorname{Mod}(B$ weak $M)=\operatorname{Mod}(B: F C I-A)$,

2) The restrictions of the additive functors $\operatorname{Hom}_{B}\left(M \otimes_{A} B,-\right)$ and $-\otimes_{E}\left(M \otimes_{A} B\right)$ form an equivalence of the full additive subcategories $\operatorname{Mod}(B: F C I-A)$ and $M o d(E: F G P-D)$ of Mod-B and Mod-E, respectively.

3) The restrictions of the additive functors

$\operatorname{Hom}_{B}\left(-, M \otimes_{A} B\right)$ and $\operatorname{Hom}_{E}\left(-, M \otimes_{A} B\right)$ form a duality of the full additive subcategories $\operatorname{Mod}(B: F C I-A)$ and $\operatorname{Mod}(E: D-F G P)$ of Mod-B and E-Mod, respectively.

\section{Proof.}

$(1) \Leftrightarrow(2)$ By Proposition 2.5.

$(1) \Leftrightarrow(3)$ With the assumption

$$
\left(M \otimes_{A} B\right) \cong \operatorname{Hom}_{A}(B, M),
$$

This is clear from Theorem 2.9 (see Remark 2.6-(2)(3)).

Corollary 2.11 Let $M$ be a right A-module such that $M$ is a finitely cogenerated injective cogenerator in Mod-A. Let $\left(M \otimes_{A} B\right) \cong \operatorname{Hom}_{A}(B, M)$ as B-modules and let $M \otimes_{A} B$ be $M$-reflexive. Then the restrictions of the additive functors $\operatorname{Hom}_{B}\left(-, M \otimes_{A} B\right)$ and

$\operatorname{Hom}_{E}\left(-, M \otimes_{A} B\right)$ form a duality of the full additive subcategories $\operatorname{Mod}(B: F C I-A)$ and $\operatorname{Mod}(E: D-F G P)$ of $M o d-B$ and E-Mod, respectively.

Corollary 2.12 Let $M$ be a right A-module. Let $\left(M \otimes_{A} B\right) \mid M$ and $\operatorname{Hom}_{A}(B, M) \cong\left(M \otimes_{A} B\right)$ as $D-B-$ bimodules. Then the following statements are equivalent for $M$. 
1) $\operatorname{Mod}(B:$ weak $M)=\operatorname{Mod}(B: F C I-A)$,

2) The restrictions of the additive functors

$\operatorname{Hom}_{B}\left(M \otimes_{A} B,-\right)$ and $-\otimes_{E}\left(M \otimes_{A} B\right)$ form an equivalence of the full additive subcategories $\operatorname{Mod}(B$ : FCI-A) and $\operatorname{Mod}(E: F G P-D)$ of Mod-B and Mod-E, respectively.

3) The restrictions of the additive functors $\operatorname{Hom}_{B}\left(-, M \otimes_{A} B\right)$ and $\operatorname{Hom}_{E}\left(-, M \otimes_{A} B\right)$ form a duality of the full additive subcategories $\operatorname{Mod}(B: F C I-A)$ and $\operatorname{Mod}(E: D-F G P)$ of Mod-B and E-Mod, respectively.

Proof. Since $\operatorname{Hom}_{A}(B, M) \cong\left(M \otimes_{A} B\right)$ as $D$ - $B$-bimodules, $V$ is $\operatorname{Hom}_{A}(B, M)$-reflexive if and only if $V$ is $M \otimes_{A} B$-reflexive. The assumption $\left(M \otimes_{A} B\right) \mid M$, implies the fact that $\left(M \otimes_{A} B\right)$ is $M$-reflexive. Hence the proof follows by Theorem 2.10.

Corollary 2.13 Let $M$ be a right A-module such that $M$ is a finitely cogenerated injective cogenerator in Mod-A. Let $\left(M \otimes_{A} B\right) \mid M$ and $\operatorname{Hom}_{A}(B, M) \cong\left(M \otimes_{A} B\right)$ as $D$-B-bimodules. Then the restrictions of the additive functors $\operatorname{Hom}_{B}\left(-, M \otimes_{A} B\right)$ and $\operatorname{Hom}_{E}\left(-, M \otimes_{A} B\right)$ form a duality of the full additive subcategories $\operatorname{Mod}(B$ : FCI-A) and $\operatorname{Mod}(E: D-F G P)$ of Mod-B and E-Mod, respectively.

\section{Acknowledgements}

This work is supported by Taif University under Project
Number (1-430-368).

\section{References}

[1] S. Al-Nofayee and S. K. Nauman, "Equivalences and Dualities between FCI and FGP Modules,” International Journal of Algebra, Vol. 3, No. 19, 2009, pp. 911-918.

[2] W. Xue, "Characterizations of Injective Cogenerators and Morita Duality via Equivalences and Dualities,” Journal of Pure \& Applied Algebra, Vol. 102, No. 1, 1995, pp. 103-107. doi:10.1016/0022-4049(95)00078-B

[3] S. K. Nauman, "Static Modules and Stable Clifford Theory," Journal of Algebra, Vol. 128, No. 2, 1990, pp. 497-509. doi:10.1016/0021-8693(90)90037-O

[4] E. Cline, "Stable Clifford Theory," Journal of Algebra, Vol. 22, No. 2, 1972, pp. 350-364. doi:10.1016/0021-8693(72)90152-4

[5] E. C. Dade, “Group-Graded Rings and Modules,” Mathematische Zeitschrift, Vol. 174, No. 3, 1980, pp. 241-262. doi:10.1007/BF01161413

[6] K. R. Fuller, "Ring Extensions and Duality," Algebra and Its Applications, Contemporary Mathematics, Vol. 259, American Mathematical Society, 2000, pp. 213-222. 